\title{
CLINICAL PATTERNS OF ORO-FACIAL INFECTIONS
}

Z.A.A. Rahman, H. Hamimah, S.S. Bunyarit.

Clinical Patterns of Oro-facial infections. Annal Dent Univ Malaya 2005; 12: 18-23.

\begin{abstract}
The aim of this retrospective study was to study the clinical patterns of oro-facial infections presented and their management (or trends of management) at the Department of Oral and Maxillofacial Surgery, Dental Faculty, University of Malaya. These included the predisposing factors, presentations and management. This study reviewed the oro-facial infection cases over 15 years. The data was obtained from case note reviews of patients using specially designed proforma. A total number of 409 samples were included in this study. Majority of the patients were generally healthy with about $6.6 \%$ having diabetes mellitus. The common presentations were pain $(47.4 \%)$, pus discharges (16.9\%) and limitation of mouth opening (12.5\%). The major site was in the submandibular region $(18.9 \%)$ followed by cheek (13.2\%). Most of the infections were from odontogenic source $(63.2 \%)$. Other sources includes cysts (15.4\%) and tumours $(6.7 \%)$. Incision and drainage were the treatment of choice performed on $57.55 \%$ of patients. Monoantimicrobial therapy was the treatment instituted in $20.8 \%$ of cases.
\end{abstract}

Key words: oro-facial infections.

\section{INTRODUCTION}

Oro-facial infections may lead to dreadful consequences. The odontogenic infections can travel downwards as far as the subphrenic space causing subphrenic abscesses (1). They may also spread into the cavernous cavity causing thrombosis of the area. Systemic spread of infections from oro-facial regions resulting in disseminated intravascular coagulation (DIVC) and septic shock had also been reported (2).

The management of oro-facial infections remains as surgical drainage, antimicrobial therapy and removal of infective sources. The choice of antimicrobials for empirical therapy include a broad spectrum usually of the penicillin group plus metronidazole targeting the anaerobic organisms (3). There is no standard regime for the antimicrobial of choice that is currently being used in the Department of Oral and Maxillofacial Surgery. The efficacy of the management of oro-facial infections in the Department had also not been documented

\section{Original Article}

Z.A.A Rahman', H Hamimah², SS Bunyarit3
1Associate Professor
Department of Oral and Maxillofacial Surgery
Faculty of Dentistry, University of Malaya
50603 Kuala Lumpur
2 Associate Professor
Department of Medical Microbiology
Faculty of Medicine, University of Malaya
50603 Kuala Lumpur
32ecturer
Faculty of Dentistry
National University of Malaysia,
Kuala Lumpur
Corresponding author: ZAA Rahman

This study is conducted to evaluate the clinical patterns of oro-facial bacterial infections and their management at the Department of Oral and Maxillofacial Surgery.

\section{MATERIALS AND METHOD}

All patients with oro-facial infections who attended the Oral and Maxillofacial Department, Faculty of Dentistry, University of Malaya from January 1984 to December 1998 were included in this retrospective study. The list of patients were taken from record of patients attendance. The patients' case notes were retrieved and reviewed using specially designed proforma. The data on patients' demography, clinical presentation, source and site of infection and treatment were collected. Patients with inadequate information and unavailable case notes were excluded from this study.

\section{RESULTS}

A total of 409 patients were included in this study. There were $258(63.1 \%)$ males and 151 (36.9\%) females. The male to female ratio was $1.7: 1$.

The incidence of oro-facial infections was highest in the 20-29 year age-group. This age group accounted for about $33 \%$ of the total cases. This was followed by the 30-39 year age group, which accounted for $20.1 \%$ of total cases (Table 1 ). The age of patients with oro-facial infections ranged from less than one year to 90 years. 
Table 1. Age, sex group distribution

\begin{tabular}{crrrrrr}
\hline Age & Number & Male & $\%$ & Female & $\%$ & Total \% \\
\hline $0-9$ & 26 & 14 & 3.4 & 12 & 2.9 & 6.3 \\
$10-19$ & 51 & 24 & 5.9 & 27 & 6.6 & 12.5 \\
$20-29$ & 135 & 102 & 24.9 & 33 & 8.1 & 33 \\
$30-39$ & 82 & 53 & 13.0 & 29 & 7.1 & 20.1 \\
$40-49$ & 45 & 24 & 5.9 & 21 & 5.1 & 11 \\
$50-59$ & 33 & 23 & 5.6 & 10 & 2.4 & 8 \\
$60-69$ & 22 & 11 & 2.7 & 11 & 2.7 & 5.4 \\
$70-79$ & 11 & 0 & 0 & 3 & 0.7 & 2.7 \\
$80-89$ & 3 & 0 & 0 & 1.7 & 0.2 & 0.7 \\
$90-99$ & 1 & 258 & 63.1 & 151 & 36.87 & 0.2 \\
\hline TOTAL & 409 & & & & & 100 \\
\hline
\end{tabular}

Table 2. Foci of oro-facial bacterial infections

\begin{tabular}{lrr}
\hline Foci Of Sepsis & Number & $\%$ \\
\hline Dento-alveolar infections & 263 & 63.2 \\
Infected cyst & 64 & 15.4 \\
Infected tumour & 28 & 6.7 \\
Infected ulcer & 20 & 4.8 \\
Cellulitis & 11 & 2.7 \\
Salivary gland infections & 8 & 1.9 \\
Non-specific inflammation & 6 & 1.4 \\
Tongue infections & 2 & 0.5 \\
Others & 14 & 3.4 \\
\hline TOTAL & 416 & 100 \\
\hline
\end{tabular}

Note: Some patients have multiple foci.

Table 3. Chief complaints

\begin{tabular}{lcc}
\hline Presentation & Number & $\%$ \\
\hline Pain & 194 & 47.4 \\
Pus discharged & 69 & 16.9 \\
Limitation to open the mouth & 53 & 13 \\
Fever & 21 & 5.1 \\
Difficulty in chewing & 16 & 3.9 \\
Dysphagia & 5 & 1.2 \\
Ulcer & 5 & 1.2 \\
Fracture of jaw & 3 & 0.7 \\
Dysphagia & 2 & 0.5 \\
Dyspnoea & 2 & 0.5 \\
Asphyxia & 2 & 0.5 \\
Unhealed wound & 2 & 0.5 \\
Drooling of saliva & 1 & 0.2 \\
Other symptoms & 34 & 8.3 \\
\hline TOTAL & 409 & 100 \\
\hline
\end{tabular}

\section{Foci of infections}

Infected dento-alveolar structures were the main diagnosis of oro-facial infections involving 263 cases $(63.2 \%)$. The dento-alveolar structures include the teeth, gums and alveolar bone. Infected cyst was the next common focus, reported in 64 patients $(15.4 \%)$. The other less common diagnoses were infected tumour, infected ulcer, cellulitis, salivary gland infection, non-specific inflammation and tongue infection (Table 2).

\section{Clinical Presentations}

The majority of patients complained of two or more symptoms. Pain was reported in 194 patients $(47.4 \%)$, and may or may not be accompanied with other signs and symptoms. The second most common complaint was pus discharge reported in 69 cases $(16.9 \%)$ followed by limitation of mouth opening reported in 53 cases $(13 \%)$ and fever, a sign which is commonly associated with infection, was reported in 21 patients $(5.1 \%)$. Difficulty in chewing was reported in 16 patients $(3.9 \%)$, while dysphagia was reported in 5 patients $(1.2 \%)$. The more serious signs and symptoms like dyspnoea and asphyxia were reported in two patients each (Table 3 ).

\section{Sites of infections}

The major sites of oro-facial infections in the head and neck was the sub-mandibular region that was seen in 79 patients $(18.9 \%)$, followed by the cheek seen in 55 patients $(13.1 \%)$. Other sites involved include the angle of mandible in 33 patients $(7.9 \%)$, the lips in 19 patients $(4.5 \%)$, the palate in 18 patients $(4.3 \%)$ and infra orbital region in 13 patients $(3.1 \%)$. The sublingual region was the least frequently involved site in oro-facial infections, reported in only 2 patients $(0.5 \%)$. The remaining 19 patients $(4.5 \%)$ had swellings which involved other sites, which were not included in this study of orofacial infections (table 4). 
Table 4. Site Of Infections

\begin{tabular}{lrr}
\hline Site Of Infections & Number & $\%$ \\
\hline Sub-mandibular & 79 & 18.9 \\
Cheek or Buccal space & 55 & 13.2 \\
Angle of mandible & 33 & 7.9 \\
Lip & 19 & 4.5 \\
Palate & 18 & 4.3 \\
Submental & 15 & 3.6 \\
Infra orbital & 13 & 3.1 \\
Tongue & 8 & 1.9 \\
Salivary gland region & 5 & 1.2 \\
Temporo mandibular joint region & 4 & 1.0 \\
Maxillary sinus & 4 & 1.0 \\
Floor of mouth & 4 & 1.0 \\
Bilateral swelling of lower jaw & 3 & 0.7 \\
Sublingual & 2 & 0.5 \\
Maxillary incisor and canine region & 37 & 8.9 \\
Maxillary premolar and molar region & 34 & 8.1 \\
Mandibular incisor and canine region & 13 & 3.1 \\
Mandibular premolar and molar region & 53 & 12.7 \\
Other sites than above & 19 & 4.5 \\
\hline TOTAL & 418 & 100 \\
\hline
\end{tabular}

Note: Some sample involves more than one area.

Table 5. Patient with systemic disease

\begin{tabular}{lrr}
\hline Systemic Disease & Number & $\%$ \\
\hline Diabetes mellitus & 27 & 6.6 \\
Kidney disease & 6 & 1.5 \\
Hypertension & 5 & 1.2 \\
Heart disease & 3 & 0.7 \\
Asthma & 3 & 0.7 \\
Leukemia & 1 & 0.2 \\
Others than above & 31 & 7.6 \\
No systemic disease & 333 & 81.4 \\
\hline TOTAL & 409 & 100 \\
\hline
\end{tabular}

Table 6. Treatment

\begin{tabular}{lrr}
\hline Treatment & Number & $\%$ \\
\hline Incision and drainage and antibiotic & 237 & 57.5 \\
Antibiotic alone & 89 & 21.6 \\
Extraction alone & 77 & 18.7 \\
Extraction with antibiotic & 9 & 2.2 \\
\hline TOTAL & 412 & 100 \\
\hline
\end{tabular}

\section{The medical history}

Surprisingly majority of patients with oro-facial infections did not have any history of underlying systemic disease $(81.4 \%)$. Twenty seven patients $(6.6 \%)$ were reported to have diabetes, six patients $(1.5 \%)$ have kidney diseases, five patients $(1.2 \%)$ have hypertension, three $(0.73 \%)$ have asthma and one patient $(0.2 \%)$ has leukemia. The 27 diabetic patients were found to have complicated oro-facial infections (Table 5). None of the patients had any history of prolonged corticosteroid therapy.

\section{Patients with allergy}

Out of 409 patients, 6 patients $(1.47 \%)$ had history of allergy to antibiotics like penicillin. Three patients were allergic to non-steroidal antiinflammatory drugs like aspirin, while another three patients had history of allergy to diabetes and heart disease drugs, sulphur group drugs and xylocaine respectively. Two patients $(0.49 \%)$ were allergic to seafood. The majority of patients $(96.33 \%)$ did not have any history of allergy. Patients with history of penicillin allergy were treated with erythromycin.

\section{Management of oro-facial infection}

Incision and drainage was the treatment of choice in the management of oro-facial infections (57.5\%). Administration of antibiotic as a single therapy was given to $21.6 \%$ of patients with orofacial infections (Table 6).

\section{Antimicrobials used in management of oro-facial infections}

Combination antimicrobial therapy to treat both aerobic and anaerobic bacteria was the treatment of choice in the management of oro-facial infections $(60.9 \%)$ in this study. 335 patients were treated with antimicrobials with or without surgical intervention (Table 6 and 7).

Metronidazole was the antimicrobial of choice to treat the anaerobic bacteria. The common antimicrobials used in combination with metronidazole were either ampicillin, amoxycillin or erythromycin. Accurate and complete data on the use of other antibiotics was not available from the case notes.

\section{Tooth associated with oro-facial infections}

Anterior teeth were the most common teeth involved in oro-facial infections (41.7\%) followed by molars $(37.4 \%)$. The least common teeth involved were bicuspid teeth 20.9\% (Table 8). Mandibular teeth were more commonly involved than maxillary teeth.

\section{Complications}

The majority of patients recovered satisfactorily with no complications. Four patients were referred for further management of their underlying systemic diseases to the University of Malaya Medical Center. 
Table 7. Usage of metronidazole in antibiotic therapy of oro-facial infection

\begin{tabular}{lcc}
\hline Antibimicrobial & $\begin{array}{c}\text { number of } \\
\text { patients }\end{array}$ & $\%$ \\
\hline Without metronidazole & 70 & 20.9 \\
Metronidazole alone & 61 & 18.2 \\
Other antimicrobials and metronidazole & 204 & 60.9 \\
\hline TOTAL & 335 & 100 \\
\hline
\end{tabular}

Table 8. Teeth associated with the oro-facial infections

\begin{tabular}{lcc}
\hline Tooth involved & number & $\%$ \\
\hline Anterior Tooth & 78 & 41.7 \\
Molar & 70 & 37.4 \\
Bicuspid & 39 & 20.9 \\
\hline TOTAL & 187 & 100 \\
\hline
\end{tabular}

\section{DISCUSSION}

A total of 409 patients, diagnosed with oro-facial infections from the year 1984 to 1998 were included in this study. The total number of samples included in this study did not reflect the overall prevalence of oro-facial infections in this department. This is because not all of the oro-facial infection cases that attended the department could be included as some of the patients' case notes were not available at the time of study. In this study, most of the oro- facial infections occurred in the age group of between 2029 years followed by patients in the 30-39 years age group. In terms of gender, there were more male than female patients with oro-facial infections. The male to female ratio was 1.7:1 (Table 1). Hunt and Meyer (4) had reported similar observations where 235 patients with oro-facial infections treated during 1978-1981 were found to occur commonly amongst males in the age group 20-29 years followed by the 30-39 years age group. The presence of underlying diseases or immuno-compromised states had been shown to influence outcome of infections. In this study it was found that out of the 409 patients, 333 patients $(81.4 \%)$ had no underlying diseases. However the 27 patients, who were diagnosed to have diabetes mellitus, were noted to have complicated oro-facial infections. Taylor et al. (5) in their study had reported that diabetes mellitus can predispose patients to severe periodontal diseases. Therefore the clinician should be aware that extensive spread of oro-facial infections could be a manifestation of an uncontrolled systemic disease like diabetes mellitus. On the other hand, diabetic patients should be made more aware of the importance of oral hygiene in preventing severe oro-facial infections. Diabetic patients are known to be more prone to developing wound infections, necrotizing fasciitis, bacteremia, pneumonia and pyelonephritis. Bacterial infections decrease insulin-mediated glucose uptake by skeletal muscle and produce whole-body insulin resistance. Acute endotoxemia and cytokine production, mostly TNF- $\alpha$ AND IL- $1 \beta$, induce insulin resistance and decreased insulin action. Oro-facial infections should be vigorously treated as they may precipitate ketosis, especially in uncontrolled diabetes.

Most of the oro-facial infections are accompanied by acute inflammatory responses. A common presenting symptom among our patients was pain. A total of 194 patients $(47.4 \%)$ in this study came with pain as the main symptom. Other more common symptoms include pus discharge (69 patients), limitation to open mouth (53 patients), difficulty in chewing (16 patients) and fever (21 patients).

Pus discharge was due to spread of infections to surrounding oral structures. The periapical odontogenic infections do not remain in the jaw bone. They may perforate the bone and discharge into the oral cavity or face. This may also explain the uncommon incident of jaw osteomyelitis.

The difficulty in chewing was due to tenderness over the involved tooth and also at the inflamed structure surrounding the infection site. Fever mostly occurs at the advanced stage of infection due to acute inflammatory response to infections, which may be localized or systemic. Bridgeman et al. (6) reported that all 107 of their patients diagnosed with oro-facial infections complained of pain while fever was noted in only $50 \%$ of those patients. Those with fever were having major oro-facial infections.

In our study, the complaints of pain and pyrexia were relatively low ,only $47.2 \%$ and $5.6 \%$ respectively. A possible explanation for these presentations could be due to the fact that our samples included those with mild and also chronic infections where pain and fever were not the main presenting symptoms. We also noted in our study two uncommon presentations, dyspnoea and asphyxia, which were seen in two cases $(0.5 \%)$ that were regarded as severe. One can only assume that the majority of patients must have come to the department early in the course of their infections to enable the clinicians to treat the infections before they could spread further to cause airway problems.

The odontogenic infections may result from dental caries, periodontal diseases, pericoronitis, dental cysts and tumors. In this study, infected dental structures were the main foci of spreading oro-facial infections, as seen in 263 cases $(63.2 \%)$. The next common foci of oro-facial infections are the jawbone cysts, which were found in 64 cases (15.4\%). In a study done by Heimdahl et al. (7), most 
of the oro-facial infections originated from necrotic pulp $(45 \%)$, infected periodontal pocket $(7.5 \%)$, postoperative procedures $(18.9 \%)$ and infected cyst $(5.7 \%)$. These can be either mild, where the infection is limited to the dental alveolar process without extending into adjacent tissues or severe where the infection extended beyond the alveolar process into adjacent anatomical spaces causing marked swelling.

A different pattern was observed by Bridgeman et al. (6) where buccal space infection $(52.6 \%)$ was the most common site followed by submandibular space infection (24\%). Another study done by Labriola et al. (8), reported that $24 \%$ of their patients presented with submandibular space infection and $20 \%$ with buccal space infection. This is almost similar to our finding.

Out of 409 patients, six patients (1.5\%) were found to have history of allergy to antibiotics, which include penicillin. This history of allergy is relatively high compared to studies done by Walters (9) and Gill et al. (10), which occurred only in $0.02 \%$ of patients prescribed with penicillin. This was probably because our data on allergies are due to several antibiotics rather than penicillin alone. The allergy was reported purely based on patient's history with no further confirmation. Information on specific antibiotic allergy was not available from the case notes.

The treatment commonly administered in the management of the 409 patients in this study was a combination of antimicrobial therapy and surgery. Two hundred and thirty-seven patients $(57.5 \%)$ had their oro-facial infections treated by incision to allow pus drainage and antimicrobials. For the remaining patients, $89(21.6 \%)$ were treated with antimicrobial only and a further 86 patients $(20.9 \%)$ were treated with tooth extraction. Similar approaches in management of oral and maxillofacial infections were echoed in a study by Bridgeman et al. (1995) where 92 of the 107 patients $(86 \%)$ were treated with the combination therapy. Drainage allows obliteration of dead spaces in cases where the abscess cavities were present. In cases where localization of pus was not apparent, the treatment used was antimicrobial alone.

Biederman and Dodson (11) in their study of paediatric patients younger than 15 years of age admitted to Grady Memorial Hospital for the management of facial infection during a five-year period, classified the infections into two groups i.e., upper face infection and lower face infection. 84\% of cases with upper face infection and 33\% of cases with lower face infection were treated with antibiotics alone. Antibiotic and surgery were carried out for $15 \%$ of cases with upper face infection and in $37 \%$ with lower face infection. Meanwhile, Bridgeman et al. (6) in their study reported that drainage was applied in 9 cases $(8.4 \%)$, extraction of teeth were performed in 7 cases $(6.5 \%)$, antibiotic alone in 30 cases $(28 \%)$, and combination of both extraction and antimicrobial therapy were reported in one case $(0.9 \%)$. In our study, the number of tooth extractions was high probably because the patients were not keen nor motivated enough to have their teeth restored or may be the teeth were grossly carious and not possible to restore.

In our study, penicillin and metronidazole were prescribed in $60.9 \%$ of the cases. A similar pattern was reported by Bridgeman et al. (6), where 81 patients $(75.7 \%)$ were reported to have received the same combination regime. Scutari and Dodson (12) reported that out of 339 adults and 143 paediatric patients $46 \%$ of upper face infections and $6 \%$ of lower face infections were treated by antibiotics alone. Surgery was utilized to $1 \%$ of upper face infections and $0.5 \%$ for lower face infections. Combination therapies were given to $53 \%$ upper face infections and $93 \%$ of lower face infections. A study done by Lewis and MacFarlane (13) reported that, short-course antimicrobial therapy has been shown to be satisfactory in the treatment of a number of common infections with the added advantage of better patient compliance, reduced accumulation of potential bacteria and reduced chance of alteration of the resident micro flora. As an example, Amoxycillin $3 \mathrm{gm}$ sachet followed by a second sachet 8 hours after first dose as opposed to phenoxymethyl penicillin $250 \mathrm{mg}$ tablet 6 hourly given for five days for the said purpose.

The study done by Lewis et al. (14) had shown that general dental practitioners were prescribing a 5-day course of penicillin, 250mg 6 hourly in $94 \%$ of patients and erythromycin 250mg 6 hourly in 73\% of patients with oro-facial infections.

Most patients diagnosed with oro-facial infections recovered uneventfully without further complications after being treated using the above treatment regimes. In this study, only four patients were admitted into the University Malaya Medical Center for further management of their underlying diseases. Eight continued to have persistent chronic infections. Many studies $(15,16,17,18)$, have reported on the complications of oro-facial infections arising from odontogenic infections, and their spread into local surrounding tissue causing local infections and also into the body systems causing morbidity and mortality. Even though mortality from oro-facial infection is possible, their incidence is low (based on isolated case reports) and this finding is comparable to this study.

\section{CONCLUSIONS}

The major source of oro-facial infections are from dento-alveolar structures. Metronidazole combined with another broad spectrum anti-microbial is the main stay for empirical antibiotic therapy for most 
oro-facial infection from odontogenic source. Surgical incisions and drainage together with antibiotic therapy are necessary for good outcome in most severe cases.

\section{REFERENCES}

1. Bounds GA. Subphrenic and mediastinitis abscess formation: A complication of Ludwig's Angina. Brit J Oral and Maxillofac Surg 1985; 23: 313-21.

2. Currie WJR, Ho V. An unexpected death associated with an acute dentoalveolar abscess - report of a case. Brit J Oral and Maxillofac Surg 1993; 31: 296-98.

3. Peterson, Ellis, Hupp, Tucker. Contemporary oral and maxillofacial surgery 4 th. Editon 2003: 343-79.

4. Hunt DE., Roger MA. Continued evolution of the microbiology of oral infections. J Am Dent Assoc 1983; Vol. 107, July, 52-5.

5. Taylor GW, Burt BA, Becker MP, Genco RJ, Shlossman M, Knowler WC, Pettitt DJ. Severe periodontitis and risk for poor glycemic control in patients with Non-insulin-dependent Diabetes Mellitus. J of Perio 1996; 67: 1085-93.

6. Bridgeman A, Wiesenfeld D, Hellyar A, Sheldon W. Major Maxillofacial infections. An evaluation of 107 cases. Aust Dent J 1995; 40:5.281-8.

7. Heimdahl A, Nord CE. Orofacial infections of odontogenic origin. Scand. J Infectious Disease. 1983; suppl.39: 86-91.

8. Labriola DJ, Mascaro J, Alpert B. The microbiologic flora of orofacial abscesses. J Oral and Maxillofac Surg. 1983; 41: 711-4.
9. Walters H. Antibiotic prophylaxis in dental surgery. Dent Update. 1997; Sept. 271-6.

10. Gill C, Pharm D. Michoelides: Dental drugs and anaphylactic reactions. Oral Surg July 1980; Vol.50, No. 1. 30-2.

11. Biederman GR, Dodson TB. Epidemiologic review of facial infections in hospitalized pediatric patients. J Oral and Maxillofac Surg 1994; 52: 1042-5.

12. Scutari P, Dodson TB. Epidemiologic review of pediatric and adult maxillofacial infections in hospitalized patients. Oral Surg Oral Med Oral Path 1996; 81: 3, 270-4.

13. Lewis AO, McGowan DA, MacFarlane TW. Short-course high-dosage amoxycillin in the treatment of acute Dento-alveolar abscess. Brit Dent J. 1986; 161: 299-302.

14. Lewis MAO, Meechan C, MacFarlane TW, Lamey P-J, Kay E. Presentation and antimicrobial treatment of acute orofacial infections in general dental practice. Brit Dent J 1989; 166: 41-5.

15. Chow AW, Roser SM, Brady FA. Orofacial odontogenic infections. Ann of Internal Med. 1978; 88: 392-402.

16. Tetsuro S, Hideki M, Toshio K, Mitsuru S. Fever of unknown origin caused by dental infection, report of a case. Oral Surg Oral Med Oral Path 1987; 64: 175-8.

17. Virolainen E, Haapaniemi J, Aitasalo K, Suonpaa J. Deep neck infections. Inter J Oral Surg. 1979; 8, 407-11.

18. Seymour RA, Steele JG. Is there a link between periodontal disease and coronary heart disease. Brit Dent J. 1998; Vol.184, No. 1 January 10. 333. 